\title{
Smart wireless system for city-gas safety management
}

\author{
J. S. Oh, J. G. Sung \& Y. D. Kim \\ Institute of Gas Safety R\&D, Korea Gas Corporation, Korea
}

\begin{abstract}
The latest industry trend seems to be a convergence around various industry fields. This paradigm is able to shift from the legacy of the service industry to a smart service environment. It is necessary to develop a wireless network and intelligent service platform in order to propagate a smart environment. However, these technologies are difficult to use in a practical real-life environment and industry facilities, because they do not consider the characteristics of the domain of industry. This paper aims to define the applicable range and criteria for a smart city-gas environment, to develop the applicable network on the target facility, and to evolve the mobile smart service platform for city-gas in order to enhance safety and reliability.
\end{abstract}

Keywords: smart service, city-gas safety management, mobile.

\section{Introduction}

The latest industry trend seems to be a convergence around various industry fields. This paradigm is able to shift from the legacy of the service industry to a smart service environment (Akydiz et al. [1]). The smart service environment is capable of innovating public service, increasing the quality of life, and enhancing business productivity (Culler and Hong [2]). It is necessary to develop a wireless network infrastructure and intelligent service platform in order to propagate a smart environment (Heinzelman et al. [3]).

However, although those technologies are progressing fast for the individual, they are insufficient for the industry environment. In order to apply them to industry, it is necessary to consider the particular characteristics and requirements of the industry environment (Oh et al. [4]). Therefore, applying smart technologies to specific industry facilities should help design the 
applicable network infrastructure which is capable of analysing the characteristics of the industry and a software platform that is able to process smart service architecture for enhancing safety and reliability (Oh et al. [5]).

Although a few technologies and studies [6-9] have introduced a smart service for the industry environment, they are concentrated on network technology. In particular, there is a gas-line network sensor system or a one wireless network in the gas industry. A gas-line network sensor system could provide an increased understanding of a distribution network, leading to cost benefits estimated to be over $\$ 10$ million annually in the USA (NETL [10]). One-wireless network is an industrial wireless network that can be designed to meet all applications requiring the flexibility and cost offered by wireless technology. It can be configured as a meshing field instruments network where each ISA 100 field instrument can communicate with more than one field instrument. However, the applicable smart industry environment is necessary not only for network technology but also for software platform technology (Honeywell [11]).

This paper focuses not only on a network but also on a mobile smart service for enhancing safety and reliability to city-gas facilities in Korea. Firstly, we describe the applicable range and criteria for the smart city-gas environment. So, we select four gas facilities, and derive measurable parameters by considering facility characteristics. In addition, three indispensable core technologies, which are reliable data transmission, minimum distance and low transmission power, are considered for our city-gas environment. Next, we account for constructing the applicable WPAN, which use, simultaneously, $2.4 \mathrm{GHz}$ and $424.7 \mathrm{MHz}$ bandwidths, into the specific pressure area and part of the test boxes, and WMAN into the valve boxes, above pipelines, and part of the test boxes. Finally, we explain the architecture of a smart service to city-gas environments, and show an outline of mobile application services for a smart city-gas portable service.

\section{Applicable range and criteria for a smart city-gas environment}

City-gas principal facilities are approximately divided into pipe, governor, valve boxes, test boxes, and so on. Measurable parameters in these facilities are classified by environmental parameters and maintenance parameters. The environmental parameters are earthquakes, ground subsidence, and so on. The others are pressure, vibration, gas leakage, and so on. This study chooses four facilities, which require preferential smart technology, and selects measurable target parameters as shown in table 1 .

The specific pressure regions aim to calculate flow using pressure and reliably transmit data in an underground closed environment. The gas governors aim to reinforce the safety of the pipe line in order to measure gas leakage, water level, current leakage and outer temperature. The test boxes of an underground pipeline aim to transmit reliable data, and the city-gas safety management service collect sensed data, predict risk, and control terminal devices. 
Table 1: $\quad$ Four facilities and parameters.

\begin{tabular}{|c|c|}
\hline Facility name & Measurable parameters \\
\hline Pressure area & Pressure \\
\hline Gas governor & Gas leakage, Water level, Current leakage \\
\hline Above pipeline & Stress, vibration \\
\hline Test box & Corrosion \\
\hline
\end{tabular}

The core of ubiquitous in the city-gas environment is reliable data transmission using wireless technology. The first point in data transmission is selection between short distance and long distance networks. In general, the specific facility such as the governor is already loaded with a wired network. Furthermore, these systems can interoperate the legacy system. According to our investigations, the specific facility is set up per distance between $1 \mathrm{~km}$ and $3 \mathrm{~km}$. Therefore, if the distance between the target and specific facility is less than $1 \mathrm{~km}$, a wireless short distance network might be applicable. Otherwise, a wireless long distance network might be appropriate, depending on costs.

The second point is the minimum distance from the closed underground environment such as gas valve boxes to near ground structures in order to transmit reliable data. Gas pipelines are laid underground along the road of a downtown area, and gas valve boxes and test boxes are formed around the gas pipe line. In these environments, communication is able to transmit from underground to the top of the structures nearby. Considering the relationship between the gas valve boxes and the structures nearby, the minimum distance is at least more than 80 meters on metros environment in Korea without attenuation data.

Other core technology is lower transmission output power in order to apply ubiquitous technology into city gas facilities. Most city-gas facilities use a battery instead of a power supply. However, we would like to use a battery for long periods without charging. In Korea, the inspection cycle is half-yearly on the basis of the city-gas business raw. Furthermore, a battery is used for at least a year without charging, because our study induces extension of the inspection cycle and enhancement of city-gas safety.

\section{Wireless network infrastructure}

\subsection{Wireless network infrastructure for communication of short distance}

In this study, the specific pressure area and test boxes are used by a wireless short distance network. According to tests for deriving an applicable frequency bandwidth, data transmission from underground to above ground seems to be less than $900 \mathrm{MHz}$, and we are able to transmit data over 100 meters with 424.7MHz. Data transmission between ground and ground uses $2.4 \mathrm{GHz}$, and the maximum distance can be over 1 kilometer. Furthermore, we have built a testbed, which consists of ten pressure monitoring areas and three gas governor 
rooms, at Gwangmyeong-si in Korea. The distance between monitoring points would be appointed at a maximum of 495 meters and a minimum of 43 meters.

Our network components are composed of transmitter, translator repeater and collector as a wireless two-tier network such as in figure 1. The transmitter senses values of a measurable parameter and transmits to the translator using a $424.7 \mathrm{MHz}$ bandwidth. The transmitter can operate in a general mode and a test mode that decides the optimum location and data reliability by checking the status of the wireless network.

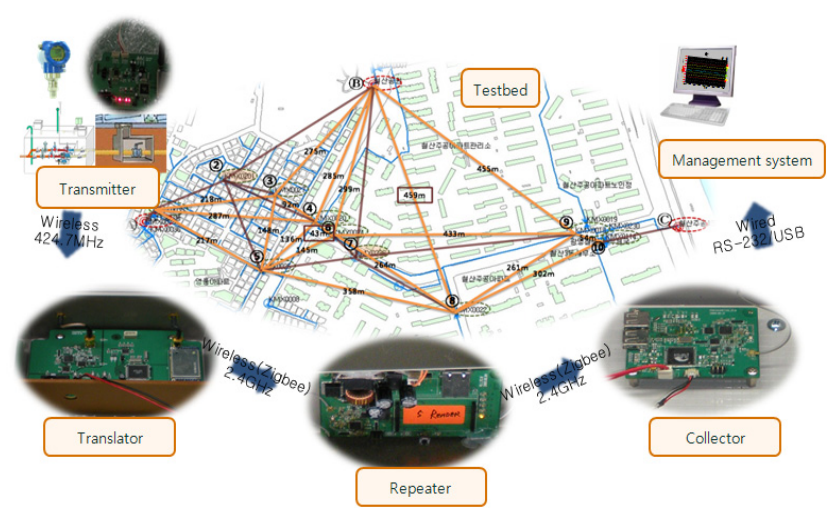

Figure 1: Data transmission of the city-gas environment in Korea.

The translator changes $424.7 \mathrm{MHz}$ into $2.4 \mathrm{GHz}$, and retransmits data to other $2.4 \mathrm{GHz}$ bandwidth components. This unit participates in a mesh network of IEEE 802.15.4a, plays the role of a bridge in a sensor network, and includes all repeater functions. It requests adding the current network component to a sink node. The relation between the transmitter and translator has a $1: 1$ or $1: \mathrm{N}$ mapping for transmitting data. A 1:1 mapping relation puts one transmitter per translator. 1:N mapping relation puts many transmitters to a translator. We apply a 1:N mapping relation because of cost and space.

The repeater receives data from another translator and repeater, and transmits data to the collector. This unit supports only an IEEE 802.15.4a wireless network, and also participates in a mesh network. The collector transmits the received data from the repeater to the management system using the Internet. This unit plays a key role as the network coordinator of the whole IEEE 802.15.4a network, and controls data transmission/reception and organization of the mesh network. The translator and repeater request the addition of the mesh network to another component of the network when first booting up, and then the collector reorganizes the mesh network.

Our sensor network uses the IEEE 802.15.4 basic MAC protocol, and a revised routing algorithm. The algorithm might be made up of a tree algorithm with a geometric progression in order to properly apply the city gas in the outside environment, and is able to generate a hierarchy according to the RSSI and the number of child nodes when the collector initiates the mesh network. In 
other words, the generation of a tree is not a basic method of IEEE 802.15.4 but the assignment of a sub-node with a geometric progression. For example, if the number of child nodes is designated as three, a two-layer hierarchy is three nodes and a three-layer hierarchy is nine nodes.

Figure 2 shows the generation order of a tree network and data transmission path. Data from the sensor node is sent to the parent node as uncast type and is sent finally to the collector. As each node might have information of the child and parent node only, it does not keep complicated path information for multihop routing. The collector recognizes relay nodes that are dropped in the network and reorganizes the mesh network.

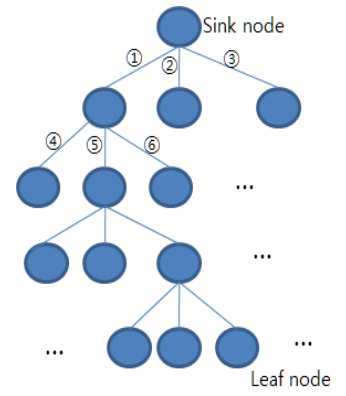

The generation order

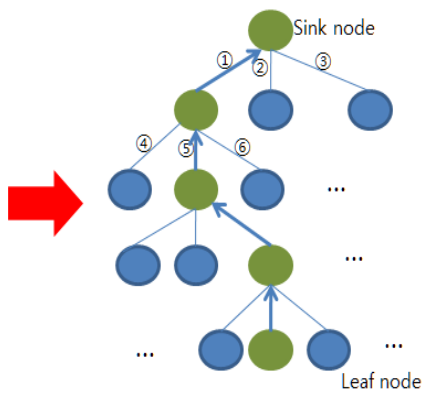

Data transmission path

Figure 2: The generation order of the network and transmission path.

\subsection{Wireless infrastructure for communication over long distances}

Gas valve boxes, above pipelines, and test boxes are used by wireless long distance networks. Gas valve boxes and test boxes have various environments such as double steel covers, and must be very reliable for data transmission. In general, the criterion of reliable data transmission is $-95 \mathrm{~dB}$. We use CDMA, which is satisfied with all cases as shown in table 2 .

Table 2: $\quad$ Sensitivity test results on CDMA.

\begin{tabular}{|c|l|l|}
\hline Facility & \multicolumn{1}{|c|}{ Status } & \multicolumn{1}{c|}{ Sensitivity } \\
\hline & Ground & $-55 \mathrm{~dB}$ \\
\hline \multirow{2}{*}{$\begin{array}{c}\text { Test boxes in } \\
\text { underground }\end{array}$} & Cover open & $-76 \mathrm{~dB}$ \\
\cline { 2 - 3 } & Cover close & $-84 \mathrm{~dB}$ \\
\hline \multirow{2}{*}{$\begin{array}{c}\text { Valve boxes in } \\
\text { underground }\end{array}$} & All cover open & $-59 \mathrm{~dB}$ \\
\cline { 2 - 3 } & Inner cover close & $-67 \mathrm{~dB}$ \\
\cline { 2 - 3 } & All cover close & $-77 \mathrm{~dB}$ \\
\hline
\end{tabular}


We design devices with low power consumption by means of changing the wake-up and sleep modes by supplying AC power around test boxes and valve boxes. The device changes the wake-up mode when an event or a specific time occurs, but it keeps sleep mode in order to minimize power consumption. Also, a poll type antenna cannot locate the exterior of a facility for a closed underground environment. Thus, this study developed a patch type antenna which can be installed on top of a box or cover. Table 3 describes the primary antenna performance, and Figure 3 shows antenna shape.

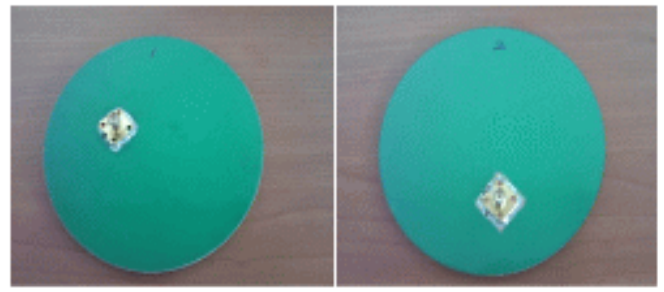

Figure 3: $\quad 850 \mathrm{MHz}$ and $2.4 \mathrm{GHz}$ antenna shape.

Table 3: $\quad$ Antenna performance.

\begin{tabular}{|l|l|}
\hline Item & Description \\
\hline Shape & Circle \\
\hline Bandwidth & $858 \mathrm{MHz}, 2.4 \mathrm{GHz}$ \\
\hline Size & Less than $\theta 800$ \\
\hline Standing wave radio & Less than $1: 1.2$ \\
\hline Efficiency & $6 \mathrm{~dB}$ \\
\hline Impedance & $\mathrm{Z}=50 \Omega$ \\
\hline
\end{tabular}

The terminal device must keep robustness to meet the conditions of table 4 on account of exposure to various environments. Our device uses a battery for about five years according to our calculation of power consumption (see table 5).

Table 4: $\quad$ Robustness condition of device.

\begin{tabular}{|l|l|}
\hline Item & Condition \\
\hline Explosion proof & Explosion-proof construction \\
\hline Water proof & IP68 \\
\hline Salt water & IEC $68-2-11 \mathrm{Ka}$ \\
\hline Vibration & $1 \mathrm{G}, 10 \sim 150 \mathrm{~Hz}, 0.1$ octave $/ \mathrm{min}$ \\
\hline Temperature & $-10^{\circ} \mathrm{C} \sim+70^{\circ} \mathrm{C}$ \\
\hline
\end{tabular}


Table 5: $\quad$ Battery test results.

\begin{tabular}{|c|c|}
\hline Item & Measured current $(\mathrm{mA})$ \\
\hline At idle & 0.02 \\
\hline At measuring & 30 \\
\hline At transmitting & $200 \sim 400$ \\
\hline A day consumption & $4.78 \mathrm{mAh}$ \\
\hline Battery & $8.5 \mathrm{Ah}$ \\
\hline Expected date & 4.87 year \\
\hline
\end{tabular}

Our research validates the accuracy of data transmission. The validation test compares the values of our device and the values of the measuring instrument on the field site. For example, table 6 shows test results with sensitivity for a protective potential device. These results can be accommodated within a permissible range.

Table 6: $\quad$ Test results on protective potential device.

\begin{tabular}{|l|l|l|l|}
\hline No & Our terminal & $\begin{array}{l}\text { Measuring } \\
\text { instrument }\end{array}$ & Sensitivity \\
\hline 1 & -1537 & -1525 & $82 \mathrm{~dB}$ \\
\hline 2 & -2350 & -2378 & $78 \mathrm{~dB}$ \\
\hline 3 & -1636 & -1653 & $81 \mathrm{~dB}$ \\
\hline 4 & -1843 & -1876 & $85 \mathrm{~dB}$ \\
\hline 5 & -2541 & -2578 & $79 \mathrm{~dB}$ \\
\hline 6 & -2134 & -2138 & $77 \mathrm{~dB}$ \\
\hline 7 & -3145 & -3121 & $83 \mathrm{~dB}$ \\
\hline 8 & -1648 & -1624 & $85 \mathrm{~dB}$ \\
\hline
\end{tabular}

\section{Smart safety management service}

A smart service for city-gas safety management consists of a network layer, adaptor layer, middleware layer, and application layer. Figure 4 shows how each adaptor interacts heterogeneous sub network resources in an adaptor layer. The adaptor receives data through various sub network interfaces, verifies data integrity, integrates the analysed data into the unified message data with referencing profile, and sends data to the middleware. Because an adaptor manages sub networks, the whole system did not influence on dropping the specific sub network.

The middleware layer supports a very large information structure in order to manage all information evolving smart information processing for autonomic risk analysis. Moreover, it provides efficient information detection with metadata and a directory service, and can deal with an interaction between middleware and services by means of supplying the open interface as shown in Figure 5. 


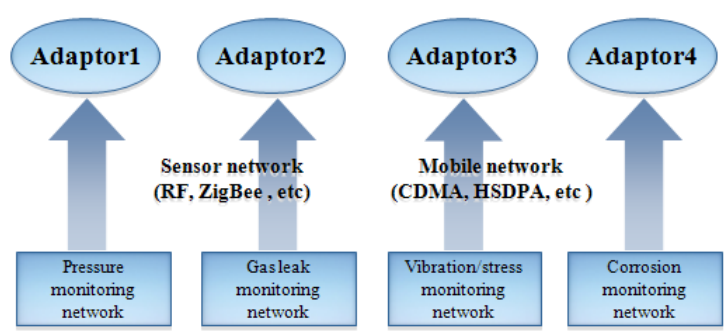

Figure 4: The architecture of the adaptor layer.

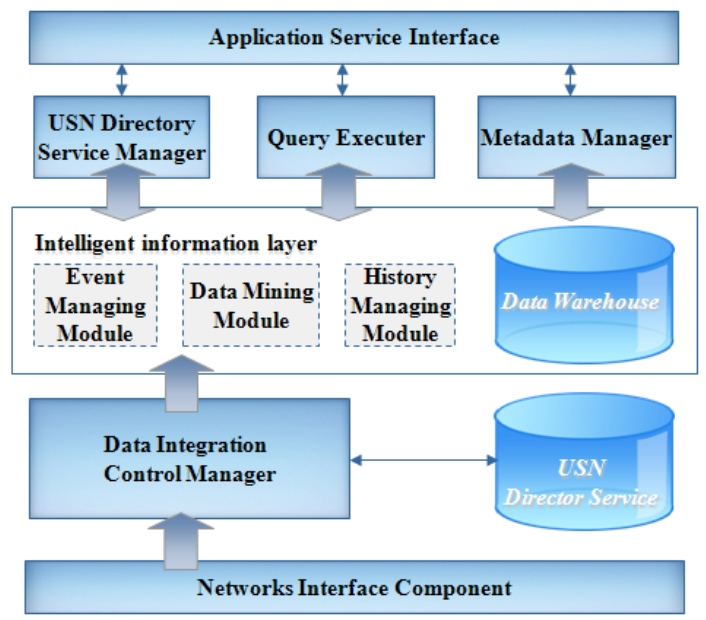

Figure 5: The architecture of middleware layers.

The application service layer processes user requirements and provides efficient results. Our application service provides both an internet and a smart portable mobile service. The safety management of the service on the internet focuses on autonomic risk analysis and efficient resource detection. The autonomic risk analysis detects an abnormal status that can be extracted by applying classification, clustering and an association algorithm using the collected information in the data warehouse. The efficient resource detection supports a fast search of a great deal of information such as meta-data, sensing data, and resource data.

The safety management of the service on a portable mobile device is more concerned with augmented reality and visualization. Figure 6 shows the outline of a portable mobile service with augmented reality. The presentation module displays data as a graph. The augmented reality module generates node location information on an LCD, and shows detailed information of the node. The data parser and validation module parse and validate the received data from a smart city-gas application service. The node management module manages meta- 


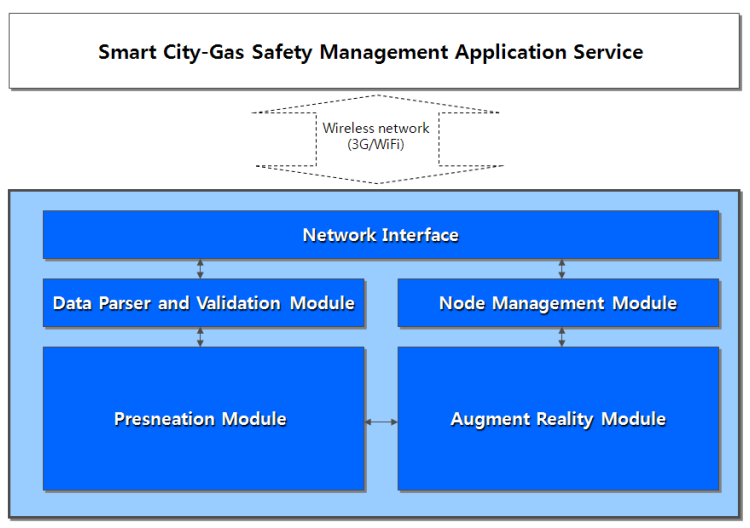

Figure 6: The architecture of a smart mobile service.

information of facilities. Furthermore, we develop advanced visualization on the mobile environment, efficient meta-information management of facilities, and connection of a smart city-gas safety management system.

\section{Conclusion}

The smart service environment is capable of innovating public service, increasing the quality of life, and enhancing business productivity. It is necessary to develop a wireless network and intelligent service platform in order to propagate a smart environment. However, these technologies are progressing fast for the individual, but they are insufficient for the industry environment. Therefore, in order to apply smart mobile technologies to specific industry facilities we must design an applicable network which is capable of analysing the industry characteristics, and a software platform that is able to process smart service architecture for enhancing safety and reliability.

Also, a smart service needs high dynamism and heterogeneity of its environment. To cope with these features, its services must rely on software middleware platforms. The smart platforms need various requirements such as an access mechanism, a control mechanism, abstraction to a heterogeneous sub network, high level modelling, and management of meta information for to the internet or mobile services.

This paper has focused on not only a network but also a mobile smart service for enhancing safety and reliability of city-gas facilities in Korea. Firstly, we have defined the applicable range and criteria for a smart city-gas environment. We have selected stress/vibration in the above pipeline, gas leakage, water level, current leakage, pressure in valve boxes, and corrosion in test boxes as measurable parameters and target facilities. In addition, our research derives three indispensable core technologies, which are reliable data transmission, minimum distance and low transmission power, for our city-gas environment.

Secondly, this paper developed network infrastructures which can be applicable from different networks on the city-gas environment in Korea. 
Because city-gas facilities with a communication device are generally set up every $1 \mathrm{~km}$ distance in a major city environment in Korea, we used a sensor network or commercial network depending on the distance between the target and gas governor. In addition, the transmission from closed to open used simultaneously bandwidths of $2.4 \mathrm{GHz}$ and $424.7 \mathrm{MHz}$ in order to meet the requirements. Furthermore, we tested the applicable performance of prototype devices and antenna in the major city environment in Korea.

Finally, our paper designed the outline of a smart service in city-gas environments. This service architecture plays an important role in order to have not influence on overall system whenever addition or drop sub network, manages all related data as the meaning information, and processes efficiently requests or results to Internet or mobile. The service on the internet focuses on autonomic risk analysis and efficient resource detection. The service on the mobile focuses more on augmented reality and visualization. The object of this paper supports a cornerstone in order to construct the framework of a smart service infrastructure for autonomic management of city-gas facilities in Korea.

\section{Acknowledgements}

This work was supported by MKIE (Ministry of Knowledge Economy) under the program of ETI (Energy Technology Innovation). This paper is a result of the "Research Group of Energy Safety for the Next Generation" (Project No 2010201010095C-21-1-000).

\section{References}

[1] Akydiz, F., Su, W., \& Sankara, Y., A Survey on Sensor Network. Journal of IEEE Communication Magazine, 40(8), pp. 102-114, 2002.

[2] Culler, D. \& Hong, W., Wireless Sensor Network. Journal of ACM Communication, 47(6), pp. 30-33, 2004.

[3] Heinzelman, W. B., Murphy, A. L., Carvalho, S. H., \& Perillo, M. A., Middleware to Support Sensor Network Applications. Journal of IEEE Network, 18(1), pp. 6-14, 2004.

[4] Oh, J. S., Park, J. S., \& Kwon, J. R., Selecting the Wireless Communication Methods for Establhishing Ubiquitous City-Gas Facilities in Korea. Lecture Notes in Computer Science, 5576, pp.823-828, 2009.

[5] Oh, J. S., Park, J. S., \& Kown, J. R., A Study on Autonomic Decision Method for Smart Gas Environments in Korea. Advances in Intelligent and Soft Computing, 72, pp. 1-9, 2010.

[6] Held, G., Data Over Wireless Networks Bluetooth, WAP, and Wireless LANs, McGraw-Hil, 2001.

[7] IEEE 802.15.4 TM, MAC and PHY Specification for Low-Rate Wireless Personal Area Networks, IEEE Computer Society, 2003.

[8] Koodli, R. S. \& Perkins, C. E., Mobile Inter-Networking with IPv6: Concepts, Principles, and Practives, Wiley-Interscience, 2007. 
[9] Shen, C., Sensor Information Networking Architecture and Applications. IEEE Personal Communications, 2(4), pp. 52-59, 2001.

[10] NETL. Oil and Natural Gas Projects (Transmission, Distribution, and Refining), http://www.netl.doe.gov.

[11] Honywell. Honeywell Process Solutions: OneWireless Network. http://hpsweb.honeywell.com/Cultures/en-US/Products/Wireless/Second GenerationWireless/default.htm. 